\title{
ANTONIO CANDIDO Y ÁNGEL RAMA, 1958. ADDENDA PARA UNA AMISTAD INTELECTUAL ${ }^{1}$
}

\author{
Hugo Herrera Pardo \\ Pontificia Universidad Católica de Valparaíso, Valparaíso, Chile \\ hugo.herrera.pardo@gmail.com
}

RESUMEN / ABSTRACT

El presente trabajo rescata un singular encuentro olvidado entre Ángel Rama y Antonio Candido, previo al verano de 1960, fecha signada como el inicio de su relación de amistad e intelectual, tanto por la crítica como por los mismos protagonistas. A partir de allí, se propone como objetivo realizar un recorrido hasta sus ensayos convenidos como centrales o canónicos para detenerse en las condiciones y modos de lectura que se fueron transformando a lo largo de ese recorrido. Travesía que es, a la vez, una especie de sinécdoque de algunas de las transformaciones más relevantes de la crítica literaria latinoamericana durante la segunda mitad del siglo pasado.

Palabras clave: Antonio Candido, Ángel Rama, crítica literaria latinoamericana, modos de lectura.

ANTONio CANDIDo AND ÁNGEL RAMA, 1958. AdDENDUM For AN INTELLECTUAL FRIENDSHIP

The present work rescues a singular forgotten encounter between Ángel Rama and Antonio Candido, prior to the summer of 1960, date marked as the beginning of their friendship and intellectual relationship, both by critics and by the protagonists themselves. From there, it is proposed as a goal to carry out a trajectory until their essays agreed as central or canonical to stop in the conditions and modes of reading that were transformed along that route. Crossing

$1 \quad$ Artículo desarrollado en el marco del Proyecto Fondecyt de Iniciación 11160086, "Genealogía de la crítica a la razón y la representación letrada latinoamericanista: debates disciplinares entre 1980-2000”, cuyo investigador responsable es el Dr. Hugo Herrera Pardo. 
that is, at the same time, a kind of synecdoche of some of the most relevant transformations of Latin American literary criticism during the second half of the last century.

KEYWORDS: Antonio Candido, Ángel Rama, Latin American literary criticism, reading modes.

Recepción: 06/11/2017

Aprobación: 19/12/2017

\section{ESPACIOS MARGINALES Y ESPACIOS PERIMIDOS}

No solo los críticos que han estudiado el vínculo intelectual y de amistad entre Antonio Candido y Ángel Rama, sino también sus mismos protagonistas, coinciden en señalar a 1960 como el año en que ambos entablan relación ${ }^{2}$. Por supuesto que la importancia de tal diálogo para el pensamiento crítico latinoamericano no solo se aprecia exclusivamente en el amplio número de trabajos que han desarrollado este vínculo. Por sobre todo también en los temas que la relación anuda: el grado de integración que a partir de la amistad entre ambos críticos comienza a proyectarse en dos importantes zonas culturales del continente como la América de habla lusa y la de habla castellana, o también la circulación de nombres, obras, conceptos y proyectos intelectuales de toda índole que tal relación transporta a lo largo de sus años. La historia, por tanto, es de sobra conocida y se puede resumir brevemente de la forma que sigue. A comienzos del citado año de 1960, Antonio Candido es invitado a dictar un curso de verano en la Universidad de la República, en Montevideo. En los meses previos a dicha invitación, el crítico brasileño ha publicado los dos volúmenes de su obra fundamental, Formação da literatura brasileira: momentos decisivos. En aquel curso de verano expone el concepto central de la obra que habría de renovar, de una manera bastante particular ${ }^{3}$ y durante

2 Ver los trabajos de Pablo Rocca (2001, 2016), Gonzalo Aguilar (2001), Grínor Rojo (2008), Pía Paganelli (2010), Eduardo Andrés Mejía Toro (2014) y José Quintão de Oliveira (2016), entre otros.

3 Lo particular de la circulación de las ideas contenidas en Formação da literatura brasileira es que ella se da sin traducción mediata del libro al español. Inclusive, su traducción es bastante tardía (Candido, Antonio. Formación de la literatura brasileña: momentos decisivos: 1750-1880. Edición, traducción, presentación y notas de Jorge Ruedas de la Serna. Facultad de Filosofía y Letras, Universidad Nacional Autónoma de México, 2014). Por tanto, el posterior éxito en la atribución de importancia y circulación a la categoría de "sistema literario" propuesta por Candido en Formaçao se debe en gran parte a este encuentro con 
los próximos dos decenios, la historiografía literaria latinoamericana; la categoría de "sistema literario". Ángel Rama se reúne personalmente con Antonio Candido por aquellos días y de tal encuentro surge una entrevista que el uruguayo publicará poco tiempo después, en la edición del 19 de febrero de 1960 del semanario Marcha y también una larga e intermitente correspondencia que se extenderá desde este momento hasta $1983^{4}$, año del fallecimiento de Rama. En los meses siguientes a aquel febrero de 1960, Ángel Rama publicará, también en el semanario dirigido por Carlos Quijano, los primeros textos en los que ensayará y empleará la categoría de "sistema literario" de Candido, para la literatura uruguaya, como "La enseñanza de la literatura" ( $\mathrm{N}^{\mathrm{o}}$ 1001, 18 de marzo de 1960) o "La construcción de una literatura" (No 1041, 30 de diciembre de 1960). Luego discutirá, expandirá y profundizará dicho uso del concepto en varios de sus trabajos importantes de las décadas del sesenta y setenta ${ }^{5}$. Así, desde su sitial en Marcha y a partir de

Rama. De hecho, en una entrevista concedida muy postrermente a Jorge Ruedas de la Serna, Candido confiesa que la primera persona que le dio importancia a las ideas contenidas en su libro fue precisamente Ángel Rama. En Brasil, a pesar de su reconocida importancia y sus múltiples reimpresiones, el libro fue, desde su misma aparición, muy resistido. Es más, cuando el texto se encaminaba ya a su cuarta edición, Candido le pidió a su editor, José de Barros Martins, que no lo volviera a publicar. Afortunadamente el libro no corrió esta suerte. Debido a una crisis financiera, tiempo después Barros Martins le vendió los derechos de la obra a la editorial Itatiaia de Belo Horizonte, la cual publicó cinco ediciones más. Posteriormente, la hija de Antonio Candido, Ana Luisa, fundaría una editorial consiguiendo los derechos de la obra y volviéndola a editar. La entrevista a la que refiero es "Antonio Candido: Cómo y por qué escribí Formação da literatura brasileira” y apareció publicada en el número 268 de Revista Casa de las Américas (julio-septiembre de 2012, pp. 117-128).

$4 \quad$ Correspondencia recientemente editada, prologada y anotada por Pablo Rocca en Un proyecto latinoamericano. Antonio Candido \& Ángel Rama, correspondencia (2016). En ella se incluye como anexo la correspondencia entre Rama y la esposa de Candido, Gilda de Mello e Souza, y como apéndice, el "Discurso de aceptación del título de Doctor Honoris Causa de la Universidad de la República" de Antonio Candido, pronunciado durante el año 2006.

Algunos de estos importantes trabajos son "Diez problemas para el novelista hispanoamericano" (1964), Rubén Dario y el modernismo (1970), "Sistema social y sistema literario en Hispanoamérica" (1975), "Un proceso autonómico: de las literaturas nacionales a la literatura latinoamericana" (1974), "Literatura y clase social" (1976), "El sistema literario de la poesía gauchesca" (1977), entre otros. Para constatar la relevancia de la categoría "sistema literario" en la obra de Ángel Rama debemos recordar un hecho que suele pasarse por alto; como él mismo lo señala en los "Agradecimientos" que se encuentran al inicio de La ciudad letrada, la primera versión de este trabajo fue una conferencia dictada en Harvard University de título "Funcionamiento del sistema literario en América Latina", durante el año 1980. Por 
su apropiación de tal categoría que busca fortalecer los vínculos entre autores y lectores en torno a una tradición particular, Rama inicia un programa de democratización literaria que recorrerá de aquí en más su carrera intelectual y que tendrá como uno de sus puntos álgidos la fundación, década y media más tarde, de la Biblioteca Ayacucho ${ }^{6}$.

No obstante lo anterior, en esta oportunidad quisiera rescatar un pequeño episodio olvidado hasta por sus propios protagonistas. Un primer encuentro entre Antonio Candido y Ángel Rama previo a ese verano de 1960. Un encuentro, eso sí, no presencial, sino que a nivel de sus firmas. Me refiero en específico a la página que ambos críticos compartieron en el periódico uruguayo Acción, el día domingo 11 de mayo de 1958. A la fecha, Ángel Rama oficiaba como crítico teatral del periódico fundado por Luis Batlle Berres -quien, por lo demás, llegaría a ser luego presidente de Uruguay, al igual que su hijo Jorge- labor que había asumido desde 1957, luego de su regreso a Montevideo tras un viaje de estudios por Europa. Sin embargo, aquel domingo de mayo de 1958, Ángel Rama no publicó una nota sobre teatro sino una reseña de un texto narrativo, en concreto sobre Coronación, la primera novela de José Donoso, nota que tituló "José Donoso. Una revelación chilena". Justo debajo de aquella nota apareció un ensayo de Antonio Candido titulado "La novela brasileña contemporánea".

Sin lugar a dudas estos dos textos ocupan un lugar absolutamente marginal y menor dentro de las respectivas obras de Candido y Rama. No es por supuesto mi intención en esta oportunidad relevarlos de aquella posición, desplazamiento que resultaría, a lo menos, forzado. Pero sí me gustaría aprovechar ese "giro" que podríamos denominar como "material" que ha sobrevolado a las humanidades y ciencias sociales desde la segunda mitad del siglo pasado, y que ha sido notorio en campos como la microhistoria, la sociología de los textos o los estudios culturales por citar solo algunos casos, "giro" en el que ha quedado patente que las textualidades o subjetividades menores y marginales contienen, a partir de la ubicación en la que han sido emplazadas, condiciones para abrir interrogantes que interpelen campos discursivos o

tanto, dicha categoría atraviesa la trayectoria como crítico de Rama, desde sus inicios en la prensa escrita hasta su etapa académica.

Para un análisis de este programa durante el periodo en que Rama ejerció como Director Literario de Marcha, se puede revisar "El tiempo de Ángel Rama (1959-1964)", uno de los capítulos que constituyen el libro de Pablo Rocca 35 años en Marcha. Mapa de la escritura en el semanario Marcha, 1939-1974. 
subjetivos más vastos. En un texto ya devenido hace bastante tiempo como clásico en nuestra disciplina, "Masa, cultura y latinoamericanismo", Julio Ramos iniciaba su análisis de "Coney Island", aquella crónica de José Martí por mucho tiempo prácticamente desatendida, señalando precisamente que "la confluencia y pugna de discursos que conforman un campo son irreductibles a los espacios perimidos, aunque canónicos de los 'grandes textos"' (71). Es desde este posicionamiento que quisiera abordar las dos notas anteriormente mencionadas, las cuales han sido relegadas al "perímetro de lo insignificante" en las respectivas obras de Candido y Rama frente a los espacios canónicos y perimidos de sus ensayísticas y desde luego también frente a sus espacios secundarios.

En este sentido, ¿qué nos puede decir este olvidado "encuentro en página" de 1958? ¿Qué modo de interpelación puede producir un hecho bibliográfico menor frente a las zonas discursivas centrales de dos críticos relevantes para la crítica latinoamericana? A partir de su palmario carácter cronológico, debido a que ambos escritos nos posicionan en un tiempo anterior al de las proposiciones teóricas, históricas y críticas que ocupan un lugar destacado en la centralidad de los corpus tanto de Candido como de Rama, contrastaré las condiciones que subyacen a estas dos notas con ciertas proposiciones contenidas en los textos considerados de mayor importancia dentro de la producción de ambos críticos. En el espacio perimido del centro, y valiéndome de consensos producidos por la institución crítica, ubicaré trabajos próximos entre sí tanto temporalmente, como en opciones teóricas y posibilidades para la apertura del campo de análisis. Me refiero a los ensayos "Literatura y subdesarrollo", "Dialéctica del malandraje" y "De cortiço a cortiço", para el caso del brasileño, junto con Transculturación narrativa en América Latina para el caso del uruguayo, además de la serie de trabajos que en su madurez ensayística le dedicó a Martí, entre ellos "Dialéctica de la modernidad en José Martí", "Indagación de la ideología en la poesía (Los dípticos seriados de Versos sencillos)" y "José Martí en el eje de la modernización poética:

\footnotetext{
Este trabajo reúne dos análisis complementarios sobre la novela de Aluísio Azevedo O Cortiço (1890): “A passagem do dois ao três" (1974) y "Literatura-Sociología: O Cortiço de Aluísio Azevedo" (1977). Cabe destacar que la primera traducción al español de "El paso del dos al tres" se registró en el tercer número de la revista Escritura (Caracas, 1977). Aquel mismo año apareció la primera versión en español de "Dialéctica del malandraje". El texto sirvió de prólogo a la novela de Manoel Antonio de Almeida Memorias de un sargento de milicias, publicada por Biblioteca Ayacucho.
} 
Whitman, Lautrémont, Rimbaud"8. En estos trabajos tan dialogantes entre sí, puede vislumbrarse un disenso con respecto a sus producciones anteriores, un esfuerzo desplegado por parte de ambos críticos para superar aspectos que hasta ese entonces resultaban dominantemente recesivos en la crítica latinoamericana, tales como el clasismo nacionalista de sus cánones, la teleología inmanente de sus trazados historiográficos o el primado de las literaturas cultas, urbanas, letradas y cosmopolitas, a partir de un examen que cuestionaba el vínculo tradicionalmente orgánico de la relación entre literatura y poder mediante un análisis basado en la coexistencia tensionante de estructuras diferentes y de temporalidades múltiples. Constituyen trabajos que, en efecto, intentan superar las paradojas democratizadoras tras el rendimiento que la categoría "sistema literario" había acumulado a lo largo de los sesenta.

En suma, la interrogante más específica que surge a partir del planteamiento anterior es: ¿qué condiciones se fueron transformando hasta devenir en sus respectivos planteamientos convenidos, a posteriori, por la institución crítica como mayormente relevantes y qué modos de lectura se fueron alterando en la concreción de esa travesía? El objetivo es así generar una perspectiva sobre la trayectoria recorrida por los autores en cuestión desde sus escritos seminales hasta sus textos centrales, trayectoria que temporalmente atraviesa aquella convulsa década de los sesenta, signada como "punto de inflexión de la modernidad tardía", a juicio de Stuart Hall, o como "los años salvajes de la teoría”, en la conocida fórmula de Manuel Asensi Pérez.

\section{OTRAS GRAFÍAS (NOTAS BIO-BIBLIOGRÁFICAS)}

De entrada, entonces, en este contraste a partir de textos menores, resultan pertinentes algunas consideraciones tanto para el caso de Rama como para el de Candido. Vayamos primero con las referidas al crítico uruguayo.

\footnotetext{
He desarrollado un análisis sobre estos textos en los trabajos "Transculturación narrativa: utopía programática modernizante" (2016) y "Modulaciones del ensayismo. Contraposiciones a partir de tres textos tempranos de Ángel Rama sobre José Marti'” (2017). Por este motivo, en el apartado final de este trabajo, me limitaré a realizar menciones sobre esta zona central de la producción ensayística de Rama en comparación a la de Candido, sin entrar en profundidad en ella, como sí lo haré con respecto a la zona central del crítico brasileño.
} 
A pesar de que Ángel Rama es en gran parte conocido por sus análisis de narrativa latinoamericana, y que de hecho comienza a cobrar relevancia continental como crítico a partir de sus intervenciones sobre el denominado -desafortunadamente- "boom" de la narrativa latinoamericana de los sesenta, el texto sobre Coronación de José Donoso constituye un hecho aislado en la producción crítica del Ángel Rama de estos años. Incluso podríamos pensar que se trata de una de sus primeras intervenciones sobre ese conjunto de escritores claramente reconocibles que con posterioridad a ese 1958 serán adscritos por el mundo editorial al "boom". Efectivamente, la idea anterior parece quedar refrendada en el cierre de sus juicios sobre la novela de Donoso en aquella nota, ya que culmina el texto señalando que lo que le parece más importante de tal propuesta es "lo que tiene de índice de una nueva situación, literaria y humana, de la creación artística, tal como la ilustra entre nosotros Juan Carlos Onetti".

Sus juicios sobre la primera novela del escritor chileno son, de hecho, el primer texto sobre narrativa que Rama publica en el periódico Acción, periódico al que se había integrado en 1957 como encargado de la sección dominical "Libros y Autores", destinada a la crítica bibliográfica y bajo la dirección de -iqué casualidad!- Juan Carlos Onetti. Al año siguiente ya se asentará en la sección de crítica teatral. De toda su producción para este periódico uruguayo, la que va desde los años 1957 a 1964, la nota sobre Coronación es una de las pocas colaboraciones en que Rama aborda objetos que no guardan relación con las artes escénicas. De hecho, en los textos que integran el primer momento de su obra, es decir aquellos escritos desde 1947 a 1955 , resulta equitativa su atención a los diversos géneros literarios ${ }^{9}$. Tras su regreso de Europa, su atención al campo teatral en específico y escénico en general concentrará una parte importante de su escritura, debido en gran medida a su trabajo en Acción, aunque cabe resaltar que su vínculo con el teatro no se restringe en lo absoluto a su colaboración en este periódico. Debemos recordar que por estos años Ángel Rama también incursiona en la

\footnotetext{
Sus textos de este periodo inicial de su producción intelectual se encuentran en las publicaciones Escritura, Asir, Clinamen, Entregas de la Licorne (en estas tres últimas participa activamente a nivel editorial), Match (también semanario, también batllista y donde también oficiará de crítico teatral), así como en el periódico El Nacional y su primer periodo de colaboración en Marcha, entre 1949 y 1950 (21 textos durante este lapso). A este momento inicial también corresponden sus primeros libros, la novela ;Oh, sombra puritana! y los ensayos La aventura intelectual de Figari y "Un estudio del Lazarillo de Tormes".
} 
actuación, la escritura teatral y hasta en el montaje de sus obras, en concreto tres, "La inundación", "Lucrecia" y "Queridos amigos", recibidas con dispar suerte por la crítica especializada montevideana ${ }^{10}$. Sus incursiones en otros autores y géneros por aquellos años -mediados de los cincuenta- habrá que encontrarlas en otras producciones de prensa escrita, como el periódico $E l$ País. Ya desde octubre de 1958, fecha en que vuelve a escribir en el semanario Marcha y se asienta en él, su atención hacia la narrativa crecerá enormemente, definiendo en gran medida su programa crítico posterior ${ }^{11}$.

Por su parte, para el caso de Antonio Candido, cabe constatar que "La novela brasileña contemporánea" no constituye su primera publicación en español. En estricto rigor, este texto representa la tercera vez que el brasileño era publicado en lengua española, aunque con solo dos textos. Me explico. "La novela brasileña contemporánea" había sido publicado por primera vez en español en aquel mismo año de 1958, pero en febrero, en el número 11 de la revista argentina Ficción. Revista-Libro bimestral ${ }^{12}$. Aunque la versión

10 Ángel Rama condensa gran parte de su experiencia en y con el teatro en su nota biográfica "El teatro y yo", aparecida en el periódico Acción el día 24 de octubre de 1958, y escrita a partir del estreno de su primera obra teatral "La inundación". Además, debe tenerse en cuenta igualmente que el año de 1953 Rama solicita formalmente el apoyo de la Facultad de Humanidades de la Universidad de la República para llevar a cabo la investigación "Origen y desarrollo del teatro nacional".

11 Ya para 1962, Ángel Rama se ha tornado uno de los emergentes especialistas en narrativa latinoamericana. Prueba de ellos es su participación en los seminarios de verano "Orientaciones de la narrativa argentina en las letras hispanoamericanas" (en la Universidad de Buenos Aires) y "Orientaciones de la narrativa sudamericana moderna" (en la Escuela Internacional de Verano de Valparaíso). Estos datos, sumados al episodio que gatilla la amistad entre Candido y Rama, revelan como necesario un estudio más específico sobre la importancia de los cursos de verano de aquellos años como instancia de circulación cultural, al menos en el Cono Sur, para el desarrollo de la crítica literaria latinoamericana de la década del sesenta.

12 Este número de la revista Ficción merece de por sí un estudio aparte, ya que significa un documento valioso en el estrechamiento de las relaciones culturales entre Brasil y la América de habla castellana. Su tabla de contenidos es relevante al menos por dos motivos. En primer lugar, el número es una auténtica antología de cuentos de la literatura brasileña. En él se incluyen los relatos "La oportunidad de Augusto Matraga" de J. Guimaraes Rosa, "Gallina ciega" de João Alphonsus, "El pavo de navidad" de Mário de Andrade, "Una noche de lluvia, o Simão, diletante de ambientes" de Ribeira Couto, "Misterio en São Cristovão" de Clarice Lispector, "El drama de la helada" de Monteiro Lobato, "La muerte de la portaestandarte" de Aníbal M. Machado, "El reloj del hospital" de Graciliano Ramos, "Estela me abrió la puerta" de Marques Rebelo, "Unos brazos" de Machado de Assis. En segundo lugar, el número presenta panorámicas introductorias en la cultura y el pensamiento brasileño, 
que apareció publicada en el periódico Acción aquel 11 de mayo de 1958 es tan solo cerca del $40 \%$ del total del ensayo y es probable que hasta haya sido tomada sin la autorización de la revista argentina, esto al menos explicaría que su referencia haya pasado totalmente inadvertida y no aparezca en las respectivas recopilaciones bibliográfícas de Antonio Candido, como las preparadas por Vinicius Dantas en Bibliografia de Antonio Candido (2002) o Márgara Russotto para el volumen antológico del crítico paulista publicado por Biblioteca Ayacucho, Crítica Radical (1991). En tanto que la primera publicación de Antonio Candido en español, al menos de la que se tiene registro hasta ahora, es el ensayo "La influencia francesa en la literatura brasileña", aparecido en el volumen Afinidades. Francia y América del Sur, publicado en Montevideo por el Servicio Francés de Información, en el año de 1946. Sobre este texto nos detendremos brevemente más adelante.

De momento se torna ineludible mencionar otras consideraciones que incluyen a ambas partes y que este olvidado encuentro contribuye a desbrozar. Una de ellas es el ámbito en el que se inscribe. De hecho y sin ir tan lejos, Antonio Candido y Ángel Rama pueden ser identificados como integrantes de aquella quizás última generación de críticos literarios (auto)formados mayormente en otras carreras humanistas dentro de la institución universitaria, siendo más bien su lugar de formación para el ejercicio crítico la prensa y las revistas culturales. Ya que a pesar de que ambos tienen estudios universitarios, siguieron allí otras disciplinas, afines a las humanidades y ciencias sociales, antes de que se formalizaran las carreras universitarias de Literatura, carreras que ellos mismos contribuirían a formar y desarrollar, en sus respectivas ciudades (o estado para la situación del brasileño, si recordamos su estadía en Assís). En el caso de Candido, formado en la Facultad de Ciencias Sociales de la Universidad de São Paulo, una de las facultades fundadoras de la universidad en 1934, el desarrollo de su carrera literaria estuvo bajo el alero de publicaciones tales como Clima, en la que fue compañero de una generación notable de críticos brasileños de diferentes artes como Décio

mediante los ensayos "El argentino y la cultura brasileña" de Gilberto Freyre, el ya citado "La novela brasileña contemporánea" de Antonio Candido, "El cuento brasileño" de Hernan Lima, "Noticia sobre el teatro brasileño" de Decio de Almeida Prado, "Manuel Bandeira y la poesía brasileña" de Alceu Amoroso Lima, "La crítica literaria en el Brasil" de Afrãnio Coutinho, "Las ideas filosóficas en el Brasil" de Cruz Costa, "El pensamiento histórico en el Brasil" de Sérgio Buarque de Holanda" y "Noticia sobre el arte moderno en el Brasil" de Lourival Gomes Machado. 
de Almeida Prado, Gilda de Moraes Rocha (la que luego sería su esposa), Lourival Gomes Machado, Paulo Emilio Salles Gomes, Ruy Coelho, entre otros, y también en el periódico Folha da Manhá, en donde sostendría la columna "Notas de crítica literária". En el caso de Rama, antes de llegar a establecerse como crítico teatral en el periódico Acción o Director Literario del semanario Marcha, había ya desde fines de los 40 fundado y participado en varias revistas culturales, tales como Asir, Entregas de la Licorne, Clinamen, entre otras. La generación de críticos que les sigue se formará mayormente bajo el alero de la universidad, siendo algunos de los ejemplos más visibles de ello los casos de Antonio Cornejo Polar o Nelson Osorio, por solo mencionar dos. Para cerrar estas ideas, un aspecto menor relacionado con esto último pero no por ello reprimible en su atención. Me refiero al uso de seudónimos en ciertas ocasiones durante su participación en la prensa escrita, para quienes luego serían dos de las firmas más significativas del pensamiento crítico y literario latinoamericano. Algunos de los seudónimos que utiliza el crítico brasileño durante su periodo en la prensa son Inácio Borges de Melo, Joaquim Carneiro y Fabricio Antunes. Mientras que algunos de los seudónimos que utiliza el crítico uruguayo durante este periodo inicial de su producción son Antonio Facal (correspondiente por cierto a uno de los recursos más reconocidos para la conformación de seudónimos, como los es la utilización del segundo nombre y segundo apellido, tal como lo haría posteriormente otro uruguayo Jorge Mario Varlotta Levrero u otro caso célebre para la literatura latinoamericana Ricardo Emilio Piglia Renzi), Martín Pérez, o el "Mercucio" con el que firmó su columna en Acción titulada "Detrás del telón", y también el uso de iniciales, como la más recurrente A.R o la más enigmática A.G (por Antonio Gundín, otro de sus seudónimos).

\section{DISTANCIAS-CERCANÍAS}

Otro aspecto a destacar de este encuentro "en página" de 1958 es cuán distantemente cerca se encontraban uno del otro. Lo cercano viene dado porque ambos utilizan las mismas categorías centrales para leer la narrativa: personaje, ambiente, estructuración, estilo. Rama aún desliza comentarios a nivel de "influencia", lo que Candido -como veremos en el siguiente apartado- había ido desplazando de su modo de lectura a partir de su singular desarrollo de la idea de "tradición". Sin embargo, distantes. Mientras Rama, en ese estilo que Candido en la primera de las cartas intercambiadas 
definirá como excelente "por su discernimiento, por la energía contenida y la economía" (39), velozmente se apura a desmarcarse de la lectura de carácter sociológico que había recibido Coronación de José Donoso en su contexto inmediato de recepción, esto es, como interpretación alegórica de la sociedad chilena, prefiriendo leerla en su lugar como "suma artística", como "esquema significativo de lo humano"13, el enfoque desarrollado por Candido en su nota es, en cambio, efectivamente sociológico. Su objetivo es presentar la organización de una secuencia histórica del desarrollo de la narrativa brasileña de la primera mitad del siglo XX con hitos bastante delimitados y caracterizando sus tendencias dominantes. De este modo, Candido excede el nivel individual autor/obra y conduce los estudios literarios al nivel de la experiencia histórica, en específico la particularidad de la experiencia local. Este paso posteriormente será fundamental para la elaboración de nuevos criterios que sirvan de base para la desarticulación y rearticulación de nuevos conjuntos literarios latinoamericanos como objetos formales de estudio, que terminaron superando los conjuntos literarios nacionales, problemática desarrollada posteriormente por Rama en artículos como "Un proceso autonómico: de las literaturas nacionales a la literatura latinoamericana" o "Sistema literario y sistema social en América Latina". Esta perspectiva característica en los panoramas esbozados por Candido, equilibrada entre lo local y lo universal, entre lo estético y lo político, entre el gusto, la erudición

13 La reseña crítica de Rama sobre Coronación es severa. Entre las virtudes de la novela expone la facilidad narrativa que presenta el autor, la que asocia a la "limpieza" de la novelística inglesa y valora asimismo la "resonancia espiritual" de la novela, junto con el encuentro tensionante entre un feliz costumbrismo y un "decidido y cruel análisis psicológico". No obstante, a su juicio, las asperezas son varias. Entre ellas se cuenta que resulta evidente que la novela no ha pasado por un proceso de corrección y estructuración posteriores, la constitución ambigua de ciertos personajes, entre ellos el mismo Andrés Abalos, mientras que a otros personajes se les concede intempestivamente un peso excesivo en el desarrollo, incorporando así "la historia innecesaria de su vida privada". En su evaluación, la novela también presenta distracciones nimias que demuestran "la falta de reacondicionamiento de la materia narrativa". Por ejemplo, la relación de Abalos y Gros se da por clausurada en determinado momento y posteriormente se los enfrenta en un diálogo, "con ellas se pretende un enriquecimiento ideológico de la obra que desfigura a ambos personajes, al dotarlos de una autoconciencia" que hasta ese momento a juicio de Rama había faltado. También el crítico uruguayo considera que el estilo mostrado por Donoso en su primera novela es "laxo, proclive a muletillas y a frases hechas". En definitiva, Rama le aconseja al autor, en aquella nota de mayo de 1958, que para una segunda edición de la novela la limpie y reduzca muchas de sus páginas. 
y el compromiso, permite un procedimiento de análisis que ha sido definido por el mismo Roberto Schwarz como una "colocação diferente dos acentos" (Seqüências Brasileiras 48), modo de lectura que así permite relativizar los esquemas universalizantes de construcción de la experiencia histórica. La cercana distancia de este encuentro de 1958, entonces, marca la primera transformación en las condiciones de lectura con la que el método crítico de Antonio Candido iniciará su repercusión en la América Latina de habla castellana. Por tanto, llegados a este punto resulta pertinente comprender la travesía de esta transformación en las condiciones de lectura. Para ello atendamos a la primera publicación de Candido en español, "La influencia francesa en la literatura brasileña".

De este texto llama la atención el arsenal léxico para llevar a cabo el análisis técnico de la panorámica presentada allí, dentro del cual sobresalen conceptos como "genio", "originalidad", "época", "movimiento", “difusión de las ideas", "asimilación", "evolución" o "intuición" (Candido nos dice que poetas románticos como Casimiro de Abreu o Fagundes Varela consiguieron "intuir el genio lírico de su pueblo"), además de la palabra clave presente en el título y organizadora del panorama presentado, la noción de "influencia". De hecho, la tesis central presentada en el texto es que la influencia francesa posibilitó el encuentro de los escritores brasileños, desde el arcadismo hasta el modernismo, con lo propio de aquel país, "sin impedir la evolución normal de nuestra vida espiritual" (62). Esta noción de "influencia" empleada en 1946 es relativizada por los planteamientos que una década más tarde servirán de presupuestos a la "Introducción" de la Formação da literatura brasileira. Justamente allí se lee que:

Más serio aún es el caso de la influencia que puede asumir sentidos variables, y requerir un tratamiento igualmente diverso. Puede, por ejemplo, aparecer como transposición directa mal asimilada, permaneciendo en la obra a la manera de un cuerpo extraño de interés crítico secundario. Puede, por otra parte, ser de tal modo incorporada a la estructura que adquiere un significado orgánico y pierde el carácter de préstamo; tomarla, entonces, como influencia, implica un prejuicio de su carácter actual, y más verdadero, de elemento propio de un conjunto orgánico (247).

La atención al carácter actual de un elemento estructurado en una obra literaria, asociado precisamente por poseer este carácter a un régimen de veridicción, señala una distancia con respecto a la proposición desarrollada 
en "La influencia francesa en la literatura brasileña" y genera las condiciones para la emergencia de aquel modo de lectura basado en una "colocação diferente dos acentos", demarcándose así los dos momentos iniciales dentro de la organización de la obra de Candido. Este tema organizativo es retomado por Gonzalo Aguilar en su ensayo "Ángel Rama y Antonio Candido: salidas del modernismo", a partir de un comentario vertido por el crítico brasileño en una entrevista de fines de los setenta. En aquella entrevista, señala Aguilar, Candido periodiza su trayectoria intelectual en tres momentos. Una primera fase "marxista-positivista que se preocupa por la causalidad y los condicionamientos y que está representada por la Introdução ao método crítico de Sílvio Romero (1945)" (75), fase a la cual de hecho podríamos adherir "La influencia francesa en la literatura brasileña". Luego, "una segunda que gira alrededor del concepto de funcionalidad según el planteo de la antropología social inglesa" (75), sobre todo de la obra de Alfred Radcliffe-Brown. Como cabría de esperarse, la obra representativa de este momento es la Formação da literatura brasileira. A continuación, se inicia "una tercera fase, a partir de Literatura e Sociedade de 1965, que se caracteriza por su interés en la estructuración, es decir, por cómo lo externo se torna interno y por cómo se estructura la estructura" (75). Finalmente, el propio Gonzalo Aguilar adiciona un cuarto momento, inaugurado por los ensayos "Literatura y subdesarrollo" y "Dialéctica del malandraje", los que propone leer en paralelo -y a los cuales podríamos agregar los trabajos en torno a la novela $O$ Cortiço de Aluízio Azevedo- debido a que estos trabajos colocan "el acento en aquellas zonas que se resisten a las formas de estructuración dominantes, instauran un linaje alternativo y exigen un nuevo aparato conceptual" (75).

Es decir que podemos interpretar cada fase dentro de la producción crítica de Candido como una alteración en el modo en que su lectura "coloca los acentos". En su primera fase, el acento es mantenido dentro de marcos occidentalizantes, mientras que en su segunda etapa éste es desplazado hacia lo local entendido como sinécdoque de lo nacional. Hacia su tercera fase, el acento se ha desplazado hasta el proceso de estructuración de las obras literarias, espacio que presenta las condiciones para comenzar a atenuar el clasismo nacionalista con que en los periodos anteriores se terminaban leyendo los textos literarios. En tanto que hacia el cuarto de los momentos distintivos el acento ya se ha descentrado del espacio hegemónico de lo nacional occidentalizante -"Parousía" o "sustancialismo logofánico" lo denominará Haroldo de Campos- para ubicarse "en aquellas zonas que se resisten a las formas de estructuración dominantes, instauran un linaje 
alternativo y exigen un nuevo aparato conceptual" (75). Por tanto, atendamos a este último desplazamiento para apreciar el trayecto recorrido por ambos críticos desde las notas que significan su encuentro en 1958.

\section{LA OPERACIÓN MALANDRO}

Existe consenso al señalar que ensayos como "Literatura y subdesarrollo", "Dialéctica del Malandraje" o "De cortiço a cortiço", consistieron en la madurez del esfuerzo crítico de Candido por explicar cómo los datos sociales se figuran en la estructura literaria. Inclusive Haroldo de Campos, uno de los mayores críticos de Formação da literatura brasileira, reconoce que el segundo de los trabajos señalados representa en cierto sentido, "la 'deslectura' deliberada" de aquel trabajo anterior, en tanto que se trata de "un segundo pensamiento proyectado con lucidez sobre su primer trazado rectilíneo y cronográfico, deslinealizándolo en pro de una nueva posibilidad de recorte inteligible del mismo espacio reorganizado ahora en una constelación diferente" (De la razón antropofágica 12). Podríamos comprender, entonces, esta "deslectura" como la inversión radical en la colocación de los acentos con respecto a las primeras de las fases críticas de la obra de Candido.

En efecto, en "Dialéctica del malandraje", y a partir de una evaluación que podemos reconocer centrada en las "convenciones" o "naturalizaciones" de selección de personajes y presentación de lugar manifiestas en la novela Memorias de un sargento de milicias de Manoel Antonio de Almeida, el pensador brasileño acaba zanjando la polémica sobre la filiación a algún género que había pasado a constituirse en el principal problema asumido por la crítica para abordar la novela. Para sostener esto, Candido una vez más desarrolla su hipótesis sobre el proceso estructurante, atisbando la función que cumple la realidad, entendida como históricamente localizada, en el fenómeno que el crítico describe, en este artículo, como "formalización o reducción estructural de los datos externos" (Crítica radical 162) ${ }^{14}$. Ahora

14 Resulta significativa en este caso, la divergencia surgida entre la filiación a algún género y un análisis históricamente localizado, este nuevo desplazamiento en la colocación de los acentos. Significativo porque, indirectamente, Candido responde a la pregunta que, casi veinte años antes, había lanzado Alfonso Reyes en su marginalia "Fragmento sobre la interpretación social de las letras iberoamericanas": "Seamos sinceros: ¿cómo negar al Pensador 
bien, en Memorias, los principios subyacentes que comunican lo social con lo literario se encuentran doblemente estratificados.

En un primer nivel, de resonancias universalizadoras, se encuentran "arquetipos válidos para la imaginación de un amplio ciclo de cultura" (164). El segundo estrato se encuentra localizado en el contexto brasileño, y se configura por una "dialéctica del orden y el desorden", en que se enmarcan las relaciones sociales de la novela, y que Candido analiza como un modo de existencia particular que es formalizado. Aquel modo de existencia particular, dato externo, lo constituye la displicencia o falta de rigor de los mecanismos estatales. Su formalización en la novela se encuentra centrada en el sistema de relaciones de los personajes, bajo la dinámica de un "orden que se comunica con un desorden que lo rodea por todos lados" (164), representación mediada por un punto de vista focal neutro asumido por el narrador, el que por ello se exime de cualquier juicio moral. El sentido profundo de esta dialéctica en la novela es el de figurar una tensión trascendente entre los esfuerzos por sistematizar, estructurar, racionalizar y sus resistencias. Una dinámica sobre todo característica de una relación metrópolis/periferia. Para Candido:

El sentido profundo de las Memórias se vincula al hecho de que ellas no se cuadraron con ninguna de las racionalizaciones ideológicas reinantes en la literatura brasileña de entonces: indianismo, nacionalismo, grandeza de sufrimiento, redención por el dolor, estilo pomposo, etc. En su estructura más íntima y en su visión latente de las cosas, este libro expresa la amplia acomodación general que disuelve extremos, interpreta el significado de la ley y del orden, manifiesta la penetración recíproca de los grupos, de las ideas, de las actitudes más dispares, creando una especie de tierra de nadie moral, donde la transgresión es apenas un matiz en la gama que desde la norma va hasta el crimen. Esta es la razón por la cual, al no manifestar estas actitudes ideológicas, el libro de Manoel Antônio de Almeida quizás

Mexicano? Pero ¿cómo medirlo, por cuanto a su función novelística, con el mismo compás que aplicamos a Balzac o a Galdós?” (Obras completas XXII 158). Esta divergencia surgida entre la filiación a alguna totalidad preestablecida - en este caso a un género- y un análisis históricamente localizado también viene a obstruir otra famosa pregunta, y su consiguiente respuesta, de la década de 1950 en los estudios históricos de las literaturas latinoamericanas, que tanto éxito tuvo, sobre todo en los Estados Unidos. Me refiero a la lanzada por Enrique Anderson Imbert en su Historia de la Literatura Hispanoamericana (1954): “AA qué delgada línea se reduciría nuestra historia, esta que ahora ofrecemos, si sólo tuviéramos en cuenta la expresión estética? Nuestras contribuciones a la literatura universal son mínimas". 
sea el único en nuestra literatura del siglo XIX que no traduce una visión de clase dominante (Crítica radical 174-175).

De este modo, por medio de la estructuración literaria de datos sociales y culturales, en un proceso entendido como continuo, Candido fractura los dos "recursos estructurales" (época y periodo) que habían subsumido a la literatura como una variable dependiente de la estructura social, modo de lectura ya bastante lejano al desplegado en "La influencia francesa en la literatura brasileña". Y no solo fractura ambos "recursos estructurales", también supera los problemas que presentaba su Formação con respecto a ellos mismos, sobre todo los referidos a que se desprendía, precisamente, "una visión de clase dominante" del conjunto de obras que acababan conformando sistema. Una importante consecuencia que se extrae del esfuerzo analítico de Candido es que, en su obra, la contradicción adquiere un valor epistemológico, mas no ontológico. Es decir, evalúa las contradicciones de la literatura latinoamericana no como una inadecuación de la producción literaria latinoamericana a las normas y valores de la cultura occidental, sino, por el contrario, por una inadecuación de estos últimos a la realidad latinoamericana. Con esto, Candido le extrae a la contradicción su rasgo de incongruencia para otorgarle un sentido, y por sobre todo un valor, de diferenciación. Esta inversión se fundamenta al asumir el vínculo sociedad-producción de pensamiento como constitutivo y constituyente y no como preestablecido, apriorístico, ya dado, en fin, como un sistema plenamente objetivo. Es más, el sistema de funcionamiento social y cultural -en otras palabras, la totalidad-propuesto por Candido se desarrolla en base a estas contradicciones. Por lo tanto, la concepción de totalidad en el autor, más que replegarse, se despliega; el examen de Memorias pone en evidencia que las diversas manifestaciones o usos de la totalidad, como géneros, movimientos o escuelas, sistemas, tradiciones, etc., son actualizadas de modo contingente por cada texto, en su proceso de producción y recepción, y no en dirección inversa, es decir, como una proyección prefigurada de aquella totalidad "aparente o falsa" -como expresaría Raymond Williams (Cultura y Materialismo 33)- hacia los textos.

Los alcances o consecuencias de estas dos operaciones descritas en el ensayo de Candido, a saber, 1) Rechazar al "pícaro" como estereotipo de Leonardo Filho sustituyéndolo por el "malandro; y 2) Explicar la estructura literaria de la novela a partir de un movimiento social e histórico concreto del contexto brasileño de la época, la dialéctica orden/desorden, son altamente significativas a efectos de romper con el discurso de la ontología moderna. 
Reconocer al "malandro" como personaje de la novela de Manoel Antonio de Almeida representa articular la literatura con una tradición que por mucho la excede (por tanto se atenúa así el primado de las literaturas cultas, urbanas, letradas y cosmopolitas en el curso genealógico de la experiencia literaria brasileña) y que la enlaza, como apunta Schwarz en el ensayo "Pressupostos, salvo engano, de "Dialética da malandragem", a una línea que viene de la colonia con el folclore de Pedro Malazarte, también un posterior género de humorismo popular e impresos cómicos y satíricos durante el periodo de la Regência. Y junto a ello algunas de las expresiones más destacadas de la tradición selectiva de la literatura brasileña, como Gregório de Matos en el periodo colonial y ambos de Andrade, Mário (en Macunaíma) y Oswald (en Serafim Ponte Grande) en el modernismo. De este modo es como podemos comprender que Candido ensaye en este trabajo el modo de lectura que consiste en colocar "el acento en aquellas zonas que se resisten a las formas de estructuración dominantes, instauran un linaje alternativo y exigen un nuevo aparato conceptual" (75), de acuerdo con las palabras de Gonzalo Aguilar.

Por tanto, a partir de lo anterior, se torna necesario divagar sobre la operación de ubicar los acentos como actividad esencial de la crítica. Con respecto a esto, para Valentín Volóshinov la realidad específica del lenguaje en tanto discurso la constituye el acontecimiento social de interacción discursiva. Y por el hecho de ocurrir en la interacción social, cada enunciado no solo posee tema y significado, sino también valoración, un "acento valorativo" en palabras de Volóshinov, el cual, y por cierto, cumple un rol fundamental; determina el ingreso de un significado referencial dado al horizonte de los hablantes. De este modo, cada cambio en la significación es, esencialmente, un cambio en el "acento valorativo". En consecuencia, la "valoración" posee una función creativa al interior de la semántica (de allí, entonces, su función esencial en la crítica). Como expone Volóshinov:

El cambio de la significación es, en el fondo, siempre una revaloración: la transferencia de una palabra determinada de un contexto valorativo al otro. La palabra o se eleva a un rango superior, o con frecuencia desciende al inferior. La separación entre el significado de una palabra y su valoración lleva irremediablemente a que el significado, desplazado de su lugar en el proceso vivo de generación social (lugar en que siempre aparece lleno de valoraciones), pasa al nivel ontológico, se convierte en una existencia ideal alejada del proceso de la generación histórica (Marxismo y filosofía del lenguaje 169). 
En este sentido, resulta oportuno mencionar que "Dialéctica del malandraje"15 apareció publicado un año antes que "Calibán" de Roberto Fernández Retamar, debido a que un altamente destacado momento de esta inversión de valores semánticos en la crítica literaria latinoamericana de fines de los sesenta y comienzos de los setenta involucra el rescate de representaciones que se interpretan como valores no occidentales ${ }^{16}$, valores que giran en torno a la figura del "Bárbaro", imagen en el proceso de occidentalización a nivel planetario producido desde el siglo XV que había pasado a ocupar una función catacrética en la representación de la otredad moderna. Aunque, por supuesto, es necesario reconocer que el "malandro" de Candido es mucho más complejo en dimensiones teóricas y críticas que el "Calibán" de Fernández Retamar. Como nos recuerda Robert Kurz, el sentido de "Bárbaro", asociado a significantes como incrédulos o paganos, hasta antes de la modernidad/ colonialidad no había estado sometido a una sistematización de carácter histórico-filosófico. Es en este momento que el concepto "Bárbaro" surgió como definición peyorativa de la humanidad antigua o contemporánea no capitalista $^{17}$. Es en esta línea de reflexión en que puede entenderse la figura del

15 El artículo apareció publicado por primera vez en la Revista do Instituto de Estudos Brasileiros $\mathrm{N}^{\circ}$ 8, São Paulo, 1970.

16 Además de las figuras del "Malandro" y el "Calibán" (en sus diferentes versiones caribeñas a lo largo de la década de los sesenta: George Lamming, Aimé Césaire, Edward Kamau Brathwaite, Fernández Retamar) podríamos sumar a este grupo el rescate del "Antropófago" oswaldiano por parte de Haroldo de Campos o hasta el mismo "transculturador narrativo" ramiano, debido a que es pensado a partir de elementos no occidentales que colisionan con elementos de procedencia externa.

17 Retomar algunos párrafos del argumento presentado por Ginés de Sepúlveda en la "Justa de Valladolid" (1550-1551) le confiere un sentido histórico concreto a estas apreciaciones de Kurz: "Pero mira cuánto se engañan y cuánto disiento yo de semejante opinión, viendo al contrario en esas mismas instituciones una prueba de la rudeza, barbarie (ruditatem barbariem) e innata servidumbre de estos hombres. Porque el tener casas y algún modo racional y alguna especie de comercio, es cosa a que la misma necesidad natural induce, y sólo sirve para probar que no son osos, ni monos y que no carecen totalmente de razón (...) por otro lado tienen de tal modo establecida su república, que nadie posee individualmente, ni una casa, ni un campo de que pueda disponer ni dejar en testamento a sus herederos, porque todo está en poder de sus señores que con impropio nombre llaman reyes, a cuyo arbitrio viven más que al suyo propio, atenidos a su voluntad y capricho y no a su libertad, y el hacer todo esto no oprimidos por la fuerza de las armas, sino de modo voluntario y espontáneo es señal ciertísima del ánimo servil y abatido de estos bárbaros [...] Tales son en suma la índole y costumbres (ingenio ac moribus) de estos hombrecillos (homunculos) tan bárbaros, incultos e inhumanos, 
"Bárbaro" como una catacresis, en tanto negación de una contemporaneidad otra. Como expresa Jed Schlosberg:

Esta negación de la contemporaneidad es producto de una evolución discursiva donde, según Mignolo, la naturaleza exótica de ese "otro" que el europeo encontró en las primeras épocas de exploración (de las Américas) se transforma de una alteridad en el espacio geográfico a una alteridad en el tiempo histórico, de manera que el "primitivo" o el "bárbaro" (el salvaje exótico) se convierte en el "otro" de la prehistoria, un humano protohistórico (o una cultura protohistórica) que solo entra en la "historia" como tal gracias a su encuentro con el "civilizador" europeo. Este aspecto "civilizador" del proyecto occidental alcanza una etapa crucial en el siglo XVIII con la representación del no-europeo colonizado como alguien que existe fuera de la historia, porque es externo al desarrollo teleológico de la "razón" -definida según la Ilustración del siglo XVIII- como principio organizador de las relaciones humanas (La crítica posoccidental y la modernidad 91).

Por lo tanto, la importancia de la revaloración o cambio en el "acento valorativo" de "Bárbaro" la constituye el hecho de que, al realizarse, no solo produce un movimiento que replantea las condiciones propias del otro signado históricamente como barbarie, sino que también implica una crítica a los fundamentos ontológicos de la modernidad occidentalocéntrica. De acuerdo con Fernando Coronil:

La crítica del locus de la modernidad desde sus márgenes crea las condiciones para una crítica intrínsecamente desestabilizadora de la propia modernidad. Deshacer la descripción de la periferia como la encarnación del atraso bárbaro, desmitifica también la autorrepresentación de Europa como la encarnación de la razón universal y el progreso histórico (El Estado mágico: naturaleza, dinero y modernidad en Venezuela 89).

Es así como el cambio en el acento valorativo como modo de lectura intentaba dar cuenta, en un nivel socio-simbólico, del desplazamiento de la representación eurocéntrica autocentrada que venía produciéndose paulatinamente en varias corrientes de pensamiento y que eclosionaría a fines de los setenta

que sabemos que así eran antes de la venida de los españoles" (Citado de Enrique Dussel, 1492: El encubrimiento del otro 71). 
en propuestas que pasarían a un lugar central en las agendas investigativas postoccidentales desde mediados de los ochenta.

\section{ASOCIACIONES VERTICALMENTE SUSPENDIDAS}

Ángel Rama despliega en sus trabajos Transculturación narrativa en América Latina y aquellos referidos a José Martí ${ }^{18}$ un objetivo de lectura similar a los atendidos por Candido en el ensayo anteriormente comentado, es decir merodear más allá de las "racionalizaciones ideológicas reinantes" en la literatura, las que habían llegado a un estado de agotamiento mediante el empleo de la categoría "sistema literario". Este es un movimiento que irrumpe como necesario en la crítica postoccidental, sobre todo en aquellas literaturas que deben sobreponerse al sustrato colonialista en que fueron históricamente asentadas. Por mencionar solo un caso comparativo, un movimiento teórico y crítico similar al "malandro" emparentado con el Pedro Malazarte de Candido podemos apreciar en un trabajo como The Signifying Monkey. A Theory of African American Literary Criticism (1988) de Henry Louis Gates Jr. En él, su autor reflexiona sobre una vasta zona de la cultura y la literatura afroamericanas que transgreden el acto de significar, mediante la metáfora de los "universos perpendiculares" y no "paralelos". De allí surge la distinción que realiza entre el Signifyin(g) negro y la Signification blanca:

What does this mean in the instance of the black homonym Signifyin( $g$ ), the shadowy revision of the white term? It means, it seems to me, that the signifier "Signification" has remained identical in spelling to its white counterpart to demonstrate, first, that a simultaneous, but negated, parallel discursive (ontological, political) universe exists within the larger white discursive universe, like the matter-andantimatter fabulations so common to science fiction. It also seems apparent that retaining the identical signifier argues strongly that the

18 A los que podríamos adherir otros trabajos de Rama ya señalados por algunos investigadores. Pienso en textos como los referidos a la poesía gauchesca, el ensayo "Las dos vanguardias" (1972), indicado por Aguilar en su artículo ya citado, o ensayos de fines de los setenta y comienzos de los ochenta dedicados a escritores como José María Arguedas, Augusto Roa Bastos o Rodolfo Walsh. Además de, por supuesto, las enormes repercusiones generadas por La ciudad letrada. 
most poignant level of black-white differences is that of meaning, of "signification" in the most literal sense. The play of doubles here occurs precisely on the axes, on the threshold or at Esu's crossroads, where black and white semantic fields collide (...) Parallel universes, then, is an inappropiate metaphor; perpendicular universes in perhaps a more accurate visual description (54).

Henry Louis Gates Jr. se vale teóricamente del concepto lacaniano de "vertically suspended associations" (55) para pensar estas significaciones que se ubican perpendicularmente en el orden de la significación dominante. Con Candido deberíamos decir "racionalizaciones ideológicas reinantes" o también un "orden que se comunica con un desorden que lo rodea por todos lados" ("dialéctica orden/desorden"). Una metáfora similar nos serviría para comprender la trayectoria de la "colocación distinta de los acentos" como modo de lectura en Antonio Candido y Ángel Rama, desde fines de la década de los cincuenta hasta mediados de la década de los setenta. Este modo de lectura se fue desplazando de manera horizontal, paralelamente, desde el polo occidental hacia el nacional para, tras saturar estas significaciones mediante la categoría "sistema literario", abrirse perpendicularmente hacia las asociaciones verticalmente suspendidas por las "racionalizaciones ideológicas reinantes".

No obstante, una diferencia radical asoma entre las propuestas de Candido y Rama, por un lado, y de Henry Louis Gates Jr., por otra. La diferencia radica en que este último autor piensa la transgresión a nivel retórico y no a nivel semántico, esto significa que en el nivel en el que indaga Gates Jr. se busca alterar el desplazamiento de los sentidos en las "rhetorical structures and strategies and thereby draws attention to the force of the signifier" (58). Mientras que a nivel semántico se produce entonces una inversión en la posición, privilegiando el término subordinado al leerlo positiva y no negativamente, pero sin embargo, se continúa pensando el fenómeno en los términos estructurales (oposición binaria) propuestos por la ontología moderna. Esta inversión revela el problema, mas no lo supera. Como apunta Stuart Hall, el aspecto negativamente crítico de la estrategia positiva/negativa es que el adicionar imágenes positivas al "ampliamente negativo repertorio del régimen dominante de la representación incrementa la diversidad" de formas de lo representado "pero no necesariamente desplaza lo negativo. Puesto que los binarismos permanecen en su lugar, el significado sigue 
estando enmarcado por ellos. La estrategia desafía a los binarismos, pero no los socava" (Sin garantías 442) ${ }^{19}$.

Aún así, trabajos como los comentados hasta acá abrieron paso a acercamientos que posteriormente cuestionaron dimensiones estructurales, tales como el histórico privilegio que los estudios humanísticos le habían asignado a la capacidad integradora de la literatura, sometiendo a examen tal sitial a partir de la relación entre el poder y la voluntad integradora característica de la subjetividad latinoamericanista, cuestionando así estrategias de legitimación vinculantes entre el Estado y la esfera cultural. Lo que de esta manera condujo a sospechar de la funcionalidad de la literatura implicada en la formación de las elites que históricamente administraron los Estados en América Latina, así como el rol de la literatura en tanto democratizador cultural, produciéndose de esta forma un desplazamiento de la centralidad ideológica que largamente le cupo. Revisitar estos trabajos desde contrapuntos establecidos con textos marginales y olvidados nos permite colocar en perspectiva este ciclo histórico de la literatura en nuestro continente y su posterior crisis.

\section{BIBLIOGRAFÍA}

Aguilar, Gonzalo. “Ángel Rama y Antonio Candido: salidas del modernismo”. Antonio Candido y los estudios latinoamericanos. Ed. Raúl Antelo. Pittsburgh: Instituto Internacional de

Literatura Iberoamericana, 2001. 71-94.

Barros-Lémez, Álvaro y Carina Blixen. Cronología y bibliografia de Ángel Rama. Montevideo:

Fundación Ángel Rama, 1986.

Candido, Antonio. "La influencia francesa en la literatura brasileña". Afinidades. Francia y América del Sur. Montevideo: Servicio Francés de Información, 1946.

"La novela brasileña contemporánea". Acción (11/V/1958): s/p.

Crítica radical. Caracas: Biblioteca Ayacucho, 1991.

O discurso e a cidade. São Paulo: Duas Cidades, 1993.

Textos de intervençao. Org. Vinicius Dantas. São Paulo: Duas Cidades, 2002.

Formação da literatura brasileira: momentos decisivos, 1750-1880. 12. ed. Rio de

Janeiro: Ouro sobre Azul; São Paulo: FAPESP, 2009.

19 En este punto, cabe resaltar que a las figuras de Esu-Elegbara y Signifying Monkey, en las que se basa Henry Louis Gates Jr. para su análisis, le subyace no solo una lógica ternaria, sino que también categorías tales como juego, ambigüedad e indeterminación. 
Formación de la literatura brasileña: momentos decisivos: 1750-1880. Edición, traducción, presentación y notas de Jorge Ruedas de la Serna. Facultad de Filosofía y Letras, Universidad Nacional Autónoma de México, 2014.

Coronil, Fernando. El Estado mágico: naturaleza, dinero y modernidad en Venezuela. Caracas: Nueva Sociedad, 2002.

Dantas, Vinicius. Bibliografia de Antonio Candido. São Paulo: Duas Cidades, 2002.

De Campos, Haroldo. De la razón antropofágica y otros ensayos. Ciudad de México: Siglo XXI, 2000.

Dussel, Enrique. 1492: El encubrimiento del otro: hacia el origen del mito de la modernidad. La Paz: UMSA. Facultad de Humanidades y Ciencias de la Educación/ Plural Editores, 1994.

Gates Jr., Henry Louis. The signifying monkey. A theory of african american literary criticism. 1988. New York: Oxford University Press, 2014.

Herrera Pardo, Hugo. "Transculturación narrativa: utopía programática modernizante”. Acta literaria 52 (2016): 81-101.

"Modulaciones del ensayismo. Contra-posiciones a partir de tres textos tempranos de Ángel Rama sobre José Martí”. Mapocho 81 (2017): 78-93.

Hall, Stuart. Sin garantías: trayectorias y problemáticas en estudios culturales. Eduardo Restrepo, Catherine Walsh y Víctor Vich (Eds.). Instituto de estudios sociales y culturales Pensar, Universidad Javeriana, Instituto de Estudios Peruanos, Universidad Andina Simón Bolívar sede Ecuador, Envión Editores, 2010.

Kurz, Robert. “A ruptura ontológica”. Um crítico na periferia do capitalismo. Reflexões sobre a obra de Roberto Schwarz. María Elisa Cevasco y Milton Ohata. Orgs. São Paulo: Companhia das Letras, 2007.

Mejía Toro, Eduardo Andrés. “Ángel Rama y Antonio Candido: la integración del Brasil en el sistema literario latinoamericano". Literatura, teoría, historia. crítica 1 (2014): 165-192.

Paganelli, Pía. "La relación intelectual de Ángel Rama y Antonio Candido: la constitución de un lenguaje crítico de cuño latinoamericano". Antíteses 5 (2010): 247-267.

Quintão de Oliveira, José. "Um diálogo ao Sul: Antonio Candido y Ángel Rama”. Línguas \& Letras 38 (2016): 44-52.

Rama, Ángel. “José Donoso. Una revelación chilena”. Acción (11/V/1958): s/p.

"La enseñanza de la literatura". Marcha 1001 (1960).

"La construcción de una literatura". Marcha 1041 (1960).

Ramos, Julio. Latinoamericanismo a contrapelo. Ensayos de Julio Ramos. Ed. Raúl Rodríguez Freire. Popayán: Universidad del Cauca, 2015.

Reyes, Alfonso. Obras completas XXII. México : Fondo de Cultura Económica, 1989.

Rocca, Pablo. "Notas sobre el diálogo intelectual Rama/Candido". Antonio Candido y los estudios latinoamericanos. Ed. Raúl Antelo. Pittsburgh: Instituto Internacional de Literatura Iberoamericana, 2001. 47-70.

Ruedas de la Serna, Jorge. "Antonio Candido: Cómo y por qué escribí Formação da literatura brasileira”. Revista Casa de las Américas 268 (2012): 117-128. 
35 años en Marcha. Mapa de la escritura en el semanario Marcha, 1939-1974. La Habana: Casa de las Américas, 2015.

Un proyecto latinoamericano. Ángel Rama \& Antonio Candido, correspondencia. Montevideo: Estuario, 2016.

Rojo, Grínor. “Ángel Rama, Antonio Candido y los conceptos de sistema y tradición en la teoría crítica latinoamericana moderna”. Discursos/Prácticas 2 (2008): 79-99.

Schlosberg, Jed. La crítica posoccidental y la modernidad. Quito: Abya Yala, 2004.

Schwarz, Roberto. Qué horas são? Ensaios. São Paulo: Companhia das Letras, 1987. Seqüencias Brasileiras. São Paulo: Companhia das Letras, 1999.

Volóshinov, Valentín. El marxismo y la filosofia del lenguaje. Buenos Aires: Godot, 2009.

Williams, Raymond. Cultura y materialismo. Buenos Aires: La marca, 2012. 